\title{
Experimenter's toolbox for learning about light and color both in the classroom and out of class
}

Gang Wang, Jacob Millspaw, Mark Masters

Gang Wang, Jacob Millspaw, Mark Masters, "Experimenter's toolbox for learning about light and color both in the classroom and out of class," Proc. SPIE 9793, Education and Training in Optics and Photonics: ETOP 2015, 97931D (8 October 2015); doi: 10.1117/12.2223108

Event: Education and Training in Optics and Photonics: ETOP 2015, 2015, Bordeaux, France 


\title{
Experimenter's Toolbox for Learning about Light and Color both in the classroom and out of class
}

\author{
Gang Wang, Jacob Millspaw and Mark Masters \\ Department of Physics, Indiana University-Purdue University Fort Wayne (IPFW), 2101 Coliseum \\ Blvd E. Fort Wayne, IN USA 46805
}

\begin{abstract}
So often in classes that teach the non-science major students are "dog and pony" shows. The students watch demonstrations, they take notes, they supposedly "absorb" the information only to forget it after the next examination. These types of classes serve only as attempts to transfer information. But what if we give the students a toolbox that provides them with the ability to make their own observations about how light works. Then, the students are empowered to plan their experiments, manipulate the simple apparatus, make their own observations, and draw their own conclusions; more closely paralleling how scientists function. To succeed at this endeavor, we carefully designed a low cost toolbox for the students. Investigations include: Additive color mixing, digital colors and filters, shadows with colors, LED spectrum and spectra, light's path, Polarization, Luminescence and Brightness, and simple optical instrumentation such as spectrometers. We created activities that give the students direction, but allow them freedom to explore and discover. Online forums/class discussion are also used to enhance their comprehension of their projects. Using this philosophy, we have had great success in both online and face to face classes.
\end{abstract}

Keywords: light and color, Optics, Undergraduate education, curriculum development, hands-on, tool-kits.

\section{INTRODUCTION}

In the state of Indiana in the United States, the science requirements are relatively weak. They consist of requiring one year of biology and one year of either chemistry or physics or a fairly unpleasant course known as integrated chemistry and physics. This means that university students often come to college without any exposure or knowledge of physics. As such, recruiting physics majors can be quite difficult and it is necessary for a physics program to have courses that can attract students who have often not thought of physics as something that might be enjoyable. Furthermore, there is simply the need to provide students with some exposure to the scientific reasoning behind physics. As such, it is necessary for physics departments to develop courses that reach such students.

One course of this type is known as Light and Color. This is a particularly popular course with enrollments of approximately 30-40 students per semester. In general, when this class has been taught face to face we have the students play with various investigations about light. However, recently, we have switched this course to a solely on-line offering. However, we believe it is critical that these on-line students have the opportunity to explore the same hands-on topics as we do in face to face sections. As such, we developed a kit that allows the students to explore the various properties of light and to learn about light through these explorations. The kits have become so popular that most students have decide to keep the kits rather than receive a refund for returning the kit. Furthermore, the kits were successful enough on line, that we started to use them in the face to face classes as well.

Education and Training in Optics and Photonics: ETOP 2015, edited by Eric Cormier, Laurent Sarger Proc. of SPIE Vol. 9793, 97931D · @ 2015 SPIE, IEEE, OSA, ICO · doi: 10.1117/12.2223108 
This required us to develop a low cost kit that is easy to use, fairly rugged and covers all the topics. The topics we identified are: additive and subtractive color mixing, associating spectra of light sources with the physiological response to the same light, shadows, ray model of light, and finally geometric optics.

\section{THE KIT}

As shown in Figure 1, the experimenter's toolkit has a wide array of items available for students to use in their investigations. These items include the light box, filters, colored objects, diffraction glasses, apertures, screens, containers for water, a flexible mirror and mirror holders, and a board for placing pins for ray tracing. It also contains fluorescent paper to use to examine the wavelength dependence of light's energy.

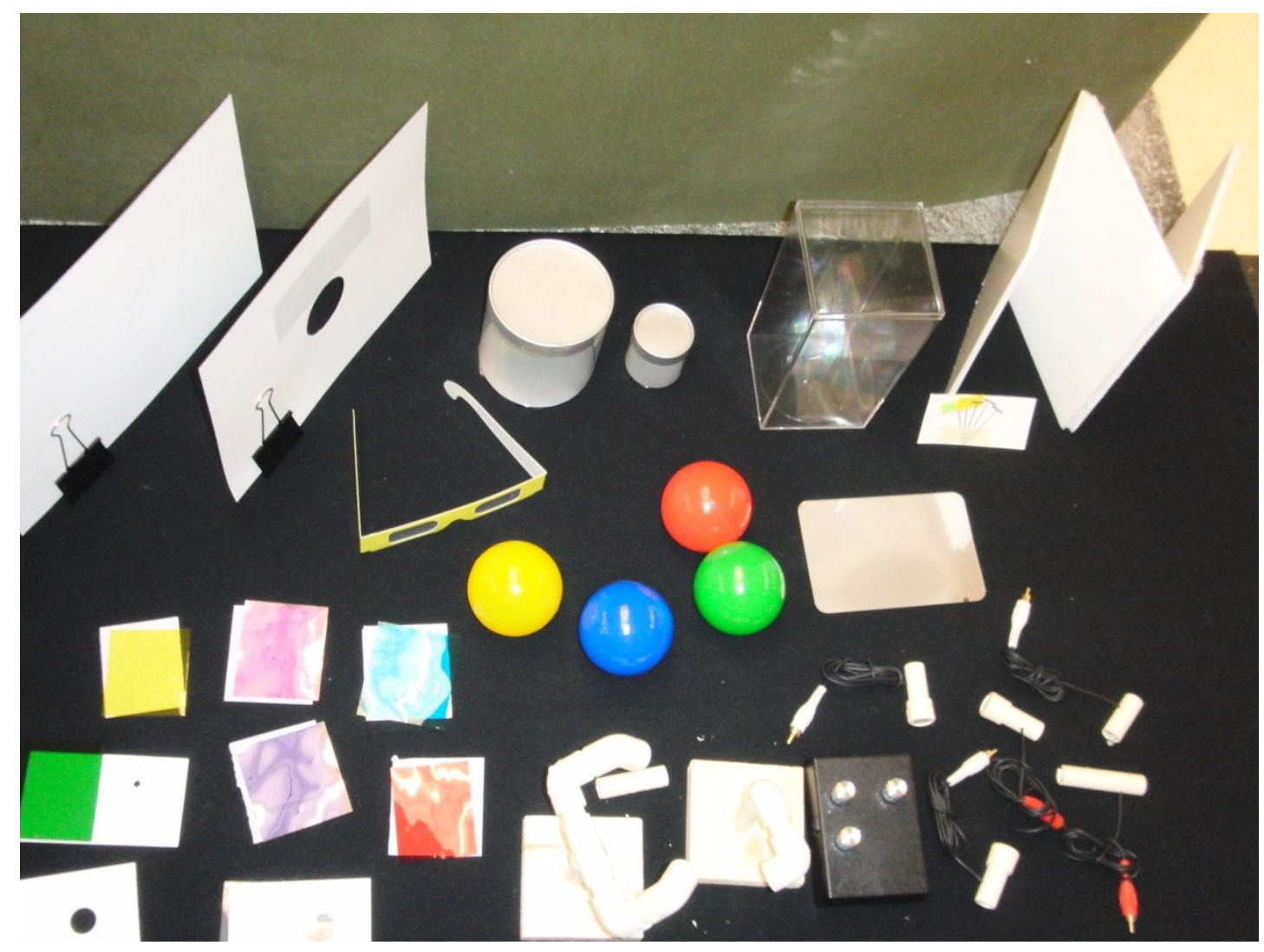

Figure 1. Photo of the supplies in the Experimenter’s Toolkit.

\section{LIGHT BOX: THE HEART OF THE KIT}

At the heart of the light kit is a light box. The light box consists of 3 separate colored LED's (Red, Green and Blue) connected with flexible wires to allow positioning them in various locations; an ultraviolet LED, an array of five yellow LED's with one red LED, and a white LED. The LED utilizes a microcontroller to control the brightness of the various channels by means of pulse width modulation. The light box has undergone a number of revisions and is currently on version 3. The various revisions have been developed for simplicity of manufacture. 
Version 3 of the light box is based on a Cypress PSoC 4 micrcontroller. A PSoC is a programmable system on a chip and contains various analog and digital capabilities. The PSoC 4 is a 32 bit ARM M0 processor with various digital and analog peripherals. One great advantage of the PSoC is the ability to use capacitive sensing. Capacitive sensing consists of buttons and "sliders" etched into the circuit board of the light box. A slider is a way to have a continuously variable control. Capsense switches have no moving parts and do not need to be soldered to the board. This is a great simplification over previous models of the light box since those required three potentiometers and switches. In addition, the buttons and sliders are incorporated into the top layer of a two layer circuit board. The PSoC and driver chip are surface mount are on the bottom layer. Thus, the circuit board becomes the lid of a 3D printed box. Figure 2 shows the board layout of the Light Box v3. Notice that there are seven buttons and a single slider. One button is the on-off button which puts the microcontroller to sleep (it draws $<1 \mu A$ ). The remaining six buttons turn on and off the various light sources. Once you turn on a light source, it remains on until toggled off. Also, while a light source is on, the slider is active allowing you to change the brightness of that light source.

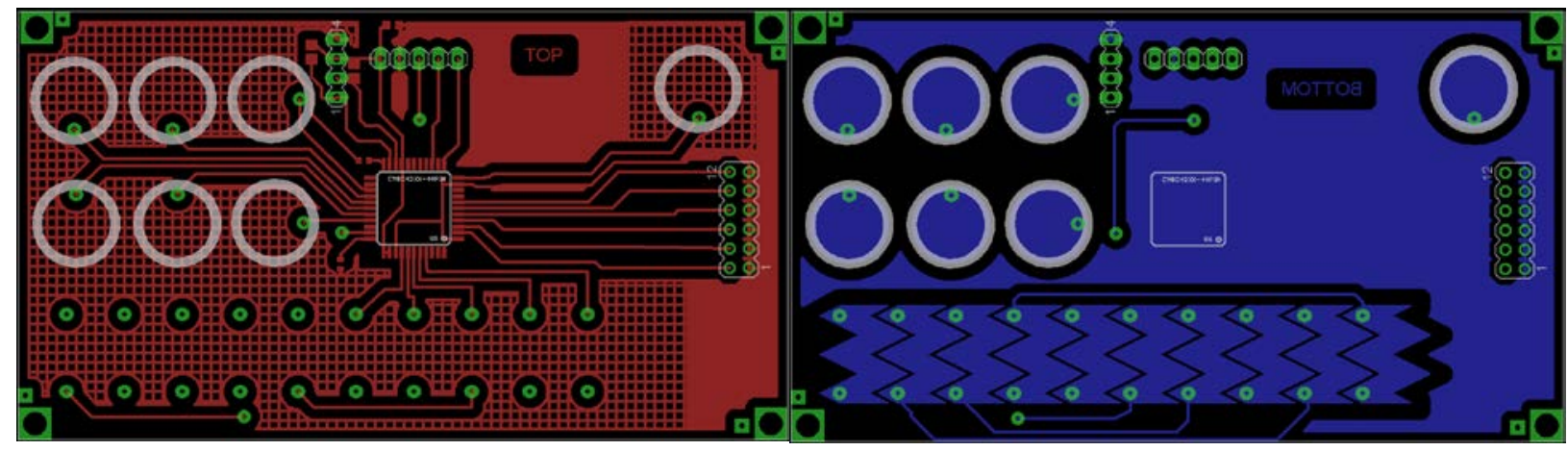

Figure 2. Version 3 board layout of prototype of the light box. Notice the capacitive slider and buttons at the top of the box (right).

The light box allows the students to explore additive color mixing. Additive color mixing causes students an amazing amount of difficulty. Even after observing the effects students often revert to subtractive mixing rules. Our pedagogy is to force students to predict what they will see, then test. The traditional color triangle is produced in Figure 3. However, we have great control over the brightness of the individual lights and thus can produce a wide gamut of colors. One challenge we often offer the students is to produce the color brown. 


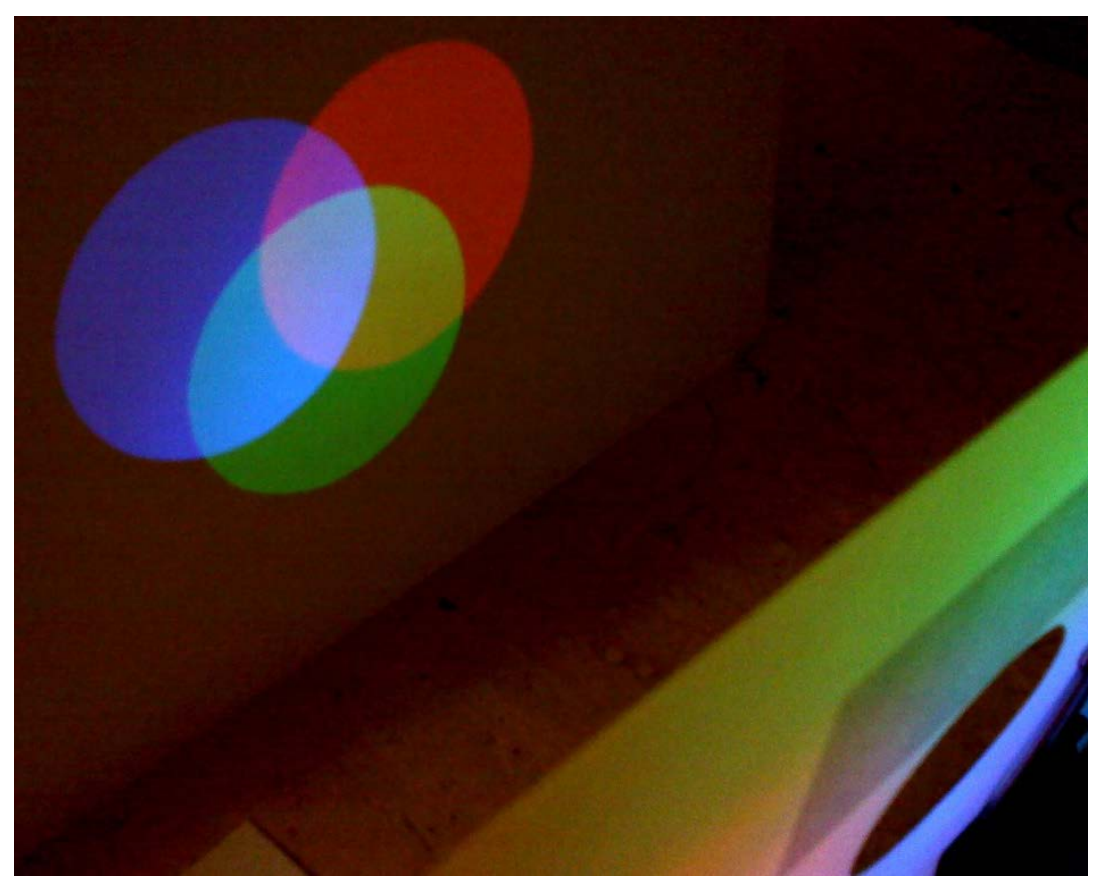

Figure 3. RGB color mixing triangle. These are produced by placing the three colored LED's close together and then having their light pass through a circular aperture.

When students make observations, it is critical that they collect evidence to support their conclusions. As such, we require students to collect data using digital cameras or cell phone cameras. These are then posted online as part of discussions or reports that are submitted for critique.

\section{SPECTRA}

Looking at the effects of overlapping different colored lights is interesting but ultimately is phenomenology. There is no explanation as to why these effects are observed. For that reason, we explore the ideas of spectrum and spectral intensity.

To have the students develop an understanding of spectra, we have them use diffractive glasses. This allows them to develop a simple comparative spectrometer. The students can, of course, simply look through the glasses which allows them to see the spectra, but it does not help them to quantify in any way. So, what we do is have the students place the glasses on a table, and kits provide an opaque mask with two apertures covered in tissue paper. These apertures allow you to illuminate the mixing screen with one or more LED's and then examine the composite spectra. Figure 4 shows the mixing screen and the experimental set up used.

The reason the mixing screen has two apertures is so that they can compare the spectra observed with the white LED and compare it with any other spectrum (see Figure 5).

Once they have this image, the students can create a spectral intensity distribution chart by directly comparing the various regions of the spectrum with the apparent brightness (see Figure 6). Because they collect the full white light spectrum simultaneously with the spectrum under comparison, the students always have a calibration. In conjunction with this, the students are also learning about waves and wavelength with its association with color. 


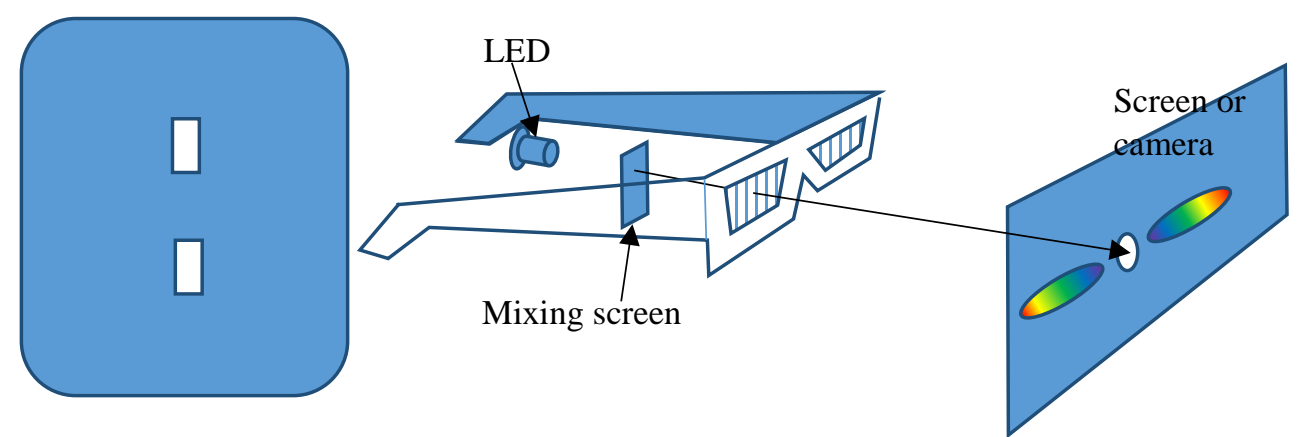

Figure 4. The mixing screen and the experimental setup the students use to produce spectra that are recorded with a digital camera.
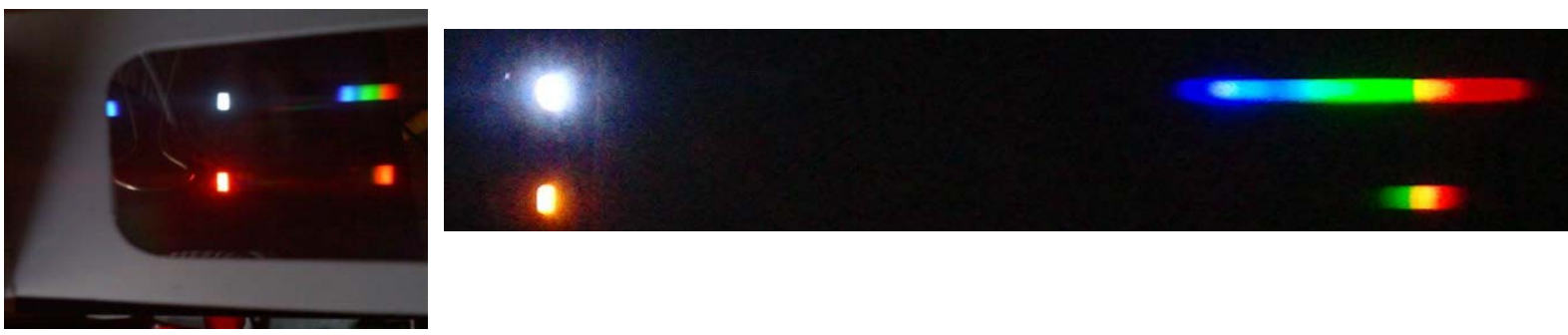

Figure 5. Two views, one showing the two apertures in the mixing screen and the two spectra. The other showing a close up of the same situation.

A)
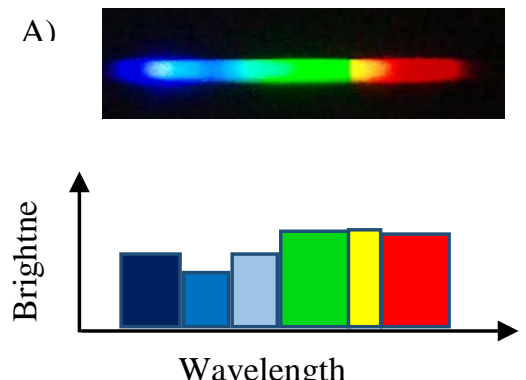

C.)

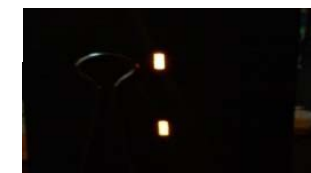

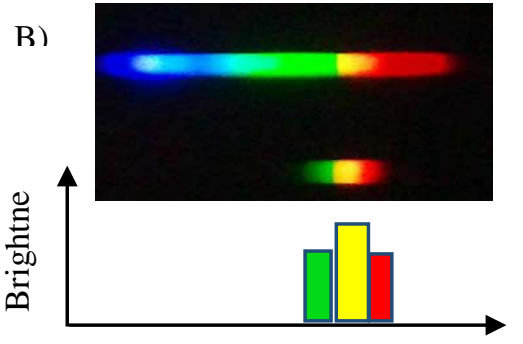

Wavelength

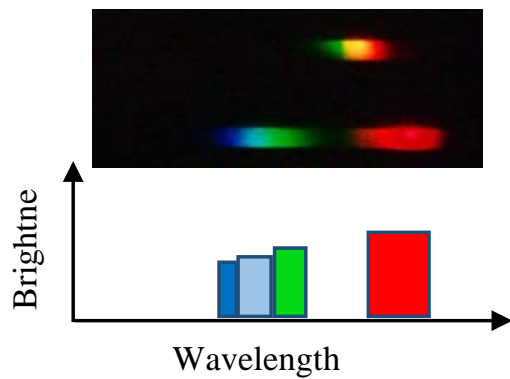

Figure 6. Spectral constructions showing the range and strength of different colors in the spectrum. A) A white LED. B) Comparison of the white LED with a yellow LED. C) Comparison of a yellow LED with a mixture of red and green LED light. 
Once the students start comparing the various spectra with physiological response, they are able to explore subtractive mixing starting with the white light and determining what colors are removed by the filters. From this, they can deduce the already somewhat familiar subtractive mixing rules.

\section{RAY OPTICS}

To start to explore ray optics we treat an LED as a point source. Using an aperture, the students can then figure out the path the light must follow. Students start with a single LED, then add a second. By moving individual LED's, they can determine how rays behave. We then introduce an "extended" source which consists of an array of 4 yellow LED's and a red LED. This is shown in Figure 7. We then move on to shadows and produce colored shadows as shown in Figure 8.

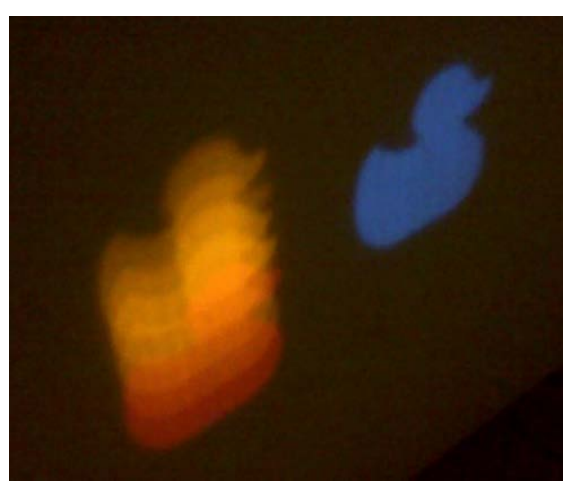

Figure 7. Comparison image of light through a duck shaped aperture. On the left, we see the image from the array of

LED's. On the right, we see the image from a single blue LED. In the array, the red LED is at the top of the array.

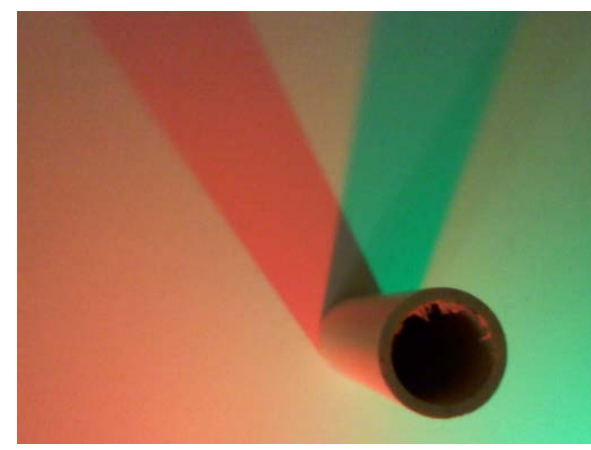

Figure 8. Colored shadows produced by using a red and a green LED that are spatially separated.

We finish off ray tracing by using water tanks and placing pins and then triangulating to enable determination of the location of a single "source" pin. This develops the idea of image formation by refraction and reflection. The most shocking discovery for students is when they form a real image of a pin with a cylindrical tank. They generally believe that they have made a mistake. 


\section{CONCLUSION}

The kits are very popular and enable the students to perform hands on investigations. There are still problems in which students need a lot of hand holding. They often feel that they need to be told precisely what to do rather than exploring and discovering. They want to get it right the first time, then be told what it means rather than thinking about the situation. Furthermore, even though the kits are engaging, the students and they seem to enjoy using them, there is still the difficulty of enforcing online participation. 